\title{
Computer Support for Social Awareness in Flexible Work
}

\author{
SUSANNE BØDKER ${ }^{1} \&$ ELLEN CHRISTIANSEN ${ }^{2}$ \\ ${ }^{1}$ Center for New Ways of Working, University of Aarhus, Aarhus, Denmark (E-mail: bodker@ \\ daimi.au.dk); ${ }^{2}$ Department of Communication, Aalborg University, Aalborg, Denmark
}

\begin{abstract}
How do we conceptualize social awareness, and what support is needed to develop and maintain social awareness in flexible work settings? The paper begins by arguing the relevance of designing for social awareness in flexible work. It points out how social awareness is suspended in the field of tension that exists between the ephemerality and continuity of social encounters, exploring ways to construct identity through relationships by means of social encounters - notably those that are accidental and unforced. We probe into this issue through design research: In particular, we present three exploratory prototyping processes in an open office setting (examining the concepts of a shared calendar, personal panels, and ambient awareness cues). Field studies, conducted in parallel, have contributed to a conceptual deconstruction of CSCW concepts, resulting in a focus on cues to relatedness, to belonging, and to care. Analyzing these three prototypes in their microcosmic usage setting results in specific recommendations for the three types of applications with respect to social awareness. The experiences indicate that the metaphors a 'shared mirror' and 'breadcrumbs' are promising foundations on which to base further design. We present these analyses and suggest that the metaphors work because of their ability to map experiences from the physical space into conceptual experiences. We conclude that social awareness in flexible work must be constructed indirectly, presenting itself as an option, rather than as a consequence of being able to overhear and oversee.
\end{abstract}

Key words: 'new' offices, ambience, design research, flexibility, social awareness

\section{Introduction}

People are increasingly working in mobile, or flexible settings. Work has become a moving target with respect to content and obligations. It is subject to constant change; in goals, in qualifications, and in partnerships. New people join groups, projects, and companies. Companies hire, fire, or close, or are taken over by others. The instability this turmoil creates in our social networks puts people under pressure, causing what Sennett calls a "corrosion of character" (Sennett, 1998). In some instances, however, change is counterbalanced by the formation of new professional networks, in addition to team- and company-based collaboration (Nardi et al., 2000a). Structures surrounding and penetrating such 'new' work arise out of mobile, networked technology and project-managed organizations. New office designs are explicitly motivated by the wish to facilitate creativity, knowledge sharing, and communication. Work is carried out across a variety of settings: offices, 
homes and airports, not to mention coffee shops and cars. Hype or not, people who may see themselves as working from a desk spend, on average, less than $50 \%$ of their working hours at their desk, and show a remarkable diversity in their working patterns (Duffy, unpublished lecture; Bjerrum and Bødker, 2003; Bjerrum and Bøgh Fangel, 2004). Hallways and moving vehicles become legitimate workspaces as work practices become more and more dispersed and mobile. Those working are always 'on-line' and able/ required to work wherever they are. At the same time, anchoring in regular work patterns and partnerships is disappearing. "Everything, everywhere", and "my office in my pocket" are visions that permeate advertisements and design for, and research on, new gadgets. Furthermore, there is a tendency for instance in computer-supported cooperative work (CSCW) - to extend design ambitions to comprise a multitude of awareness functions and services in the metaphor of a single interface, such as a desktop or the baby-face of a cellphone. The question is, however: How do we decide which structures to support? What is the relationship between the time-place structure and the structure supporting social awareness? Clearly workers are under a lot of pressure to be constantly "on-line". The intuitive way of handing over experience in the workplace becomes more difficult as people are deprived of stable locations and fixed working hours and partnerships. The 'legitimate peripheral participation,' which Lave and Wenger (1991) have described as the intuitive way of learning in a workplace, is under assault. Accordingly, designers of CSCW have to compensate for the lack of time and place fixation while supporting learning, participation, and social awareness. Again the question is: Which alternative structures should be supported?

\subsection{BASIC ASSUMPTIONS AND CONDITIONS}

In recent years we have participated in several projects on the 'New office'; the "modern" open office space (for an overview, see Bjerrum and Bødker, 2003). Social awareness and the "background" maintenance of a social (and perhaps virtual) community have moved to the forefront in our field of interest. A recurring concern in our case studies is that the 'New office,' which was intended to support learning, lacks places to dwell, and to return to, and places to meet coincidentally, and lacks shared artefacts and possibilities for leaving traces of current and past activities. These deficiencies stem from a combination of problematic architecture and insufficient technology and, not least, from inadequate design processes: People's actual needs in an organization are rarely considered, and people are seldom involved in the design and introduction processes (Bjerrum and Bødker, 2003).

To illustrate these findings, let us look at four examples:

1. In a start-up company (Christiansen, 2001), groups starting up a project will draw on previous habits, and will try to spend time together and 
talk while making as much use as possible of their shared physical space. Physical boards and electronic media are important, but only after the group members have established a common ground in the immediate physical space do they allow themselves to work apart.

2. An engineering company (Bjerrum and Bødker, 2003) occupies two large open floors with no room dividers or partitions. Engineers move their desks and reconfigure their shared space as and when new projects are established. This works well for the projects, but due to the lack of wall space and the frequent moves, people complain that it is difficult to know about and keep track of the various projects, and to locate people in general and the expertise needed for one's own project in particular.

3. In a media company (Aaløkke et al., 2005), many employees work in a large, open space where people walk to and fro, and where the soundscape is dominated by large and small meetings, and ringing telephones. Despite this very noisy environment, people are happy with the arrangement because they consider participating in meetings and communicating as a major part of their job. This stands in contrast to many other organizations with a much lower sound level where people tend to hush each other. They consider their individual tasks to be the most important element, and they have no good ways of demonstrating availability to each other (Aaløkke and Bjerrum, 2005).

4. A public agency (Bjerrum and Bødker, 2003) applies a hotel concept where people "hot-desk". Their office building is characterized by a very restrictive "exhibition" policy. People are not allowed to hang sketches or pictures on the walls. One collective rule is a "clean desk" that requires the workers to clean up their desk every day. This consistently prevents employees from leaving personal items, or "stuff", around that might show what they do.

These case studies point towards some general concerns: Workers in the flexible setting often lack a sense of whether colleagues are in or out of the office, and whether they are working from home or from somewhere else. It is difficult to know when and where to get in touch with co-workers. They lack a sense of co-presence that tells them when the others are available, and even where to leave a message if necessary. They often find it difficult to take possession of a space and construct a "home" where they work. And perhaps most importantly: Workers in the flexible setting are never sure who it is important for them to bond with, because co-workers can be transferred or replaced from one day to the next.

In CSCW, social awareness has been defined as follows:

awareness about the social situation of the members [our emphasis] i.e., awareness about what they are doing, if they are talking to someone, if they can be disturbed etc. In our everyday work, social awareness is a key element. 
We gather continuously information about our colleagues and act accordingly. If they listen, we will talk, if they are not here, we might phone them or leave a note. If they are in the right mood, we start a discussion, if not, we postpone it. (Tollmar et al., 1996)

Such a definition stresses the structural and functional relationships of communication: attention, turn-taking, and so on. Even though the CSCW literature contains a rich corpus of case studies and designs that address awareness in general, for instance through specific technologies (video, instant messaging, and e-mail), it seems that most technologies and prototypes focus on reporting and recording. This preoccupation has led to some concern for a potentially unhealthy transformation of social competencies, as voiced by Grudin (2002). Grudin's message is that the prototypicality (in terms of ephemerality, being 'first of its kind' and 'in the making') of social encounters is jeopardized if - by means of IT - the 'here - and now' is substituted with 'everywhere - and forever'; in other words, it is jeopardized if information about our activities is stored and made available outside the social context these activities were originally intended for, and perhaps made available long after they took place. The ephemerality of face-to-face meetings, the privacy of novice experiments (and failures), and the opportunity of staying on the periphery of social gatherings may be rendered impossible by the ubiquitous technology surrounding us today. This is because social presence is constantly mediated by means of explicit accounting - like tapes from surveillance cameras that are kept indefinitely.

Grudin does not relate his concern for the preservation of ephemerality of social encounters directly to social awareness. We find, however, that from the point of view of how experience is transferred from person to person, and from group to group, there is an intriguing tension between ephemerality and awareness. A tension that is pushed to the limit when it comes to social awareness.

Sociology, since Schutz, has emphasized how the social aspect is interwoven with tradition, that is, with the experience of continuity. It is because we know that we can reiterate and return to what is well known that we are able to embrace the unknown (Schutz, 1962). Strauss (1993) has deepened Schutz's phenomenology of how humans find the courage to march into the unknown: The experience of recurrence and patterns serve as 'breadcrumb' indicators of a way back. Strauss (ibid.) introduces the term 'trajectory' for the process through which traces grow into cues, which grow into even more stable structures that in turn find a local, situated form, which Strauss has termed 'locales'. In parallel to this way of describing the formation of tradition, we suggest that awareness, as moment-to-moment attention, depends on ephemerality. This is why Grudin's concern is so important: In order to stay aware of other people, you have to sense their presence as somewhat 
ephemeral, potentially disappearing. If their presence becomes too habitual, you tend to take them for granted, and you cease to pay attention. On the other hand, if you do not come across them at all, you may forget they are there. In order to stay socially aware, it seems necessary to have people within reach - not always, but at least occasionally. This tension between the ephemerality and continuity of social encounters, and likewise the need to construct identity through relationships by means of social encounters, is not generally part of the definition of social awareness in CSCW.

Reviewing the CSCW literature we have found that attempting to address the elusiveness of the social aspect in design experiments almost inevitably ends up in some sort of non-elusive, functional design. This is the case despite the fact that reading social structure from the way people go about using mundane artefacts has been a very successful method in CSCW. Activity theory adds to the examination of the many mediators of human relationships by pointing to intentional and motivational aspects of work (Engeström, 1987; Bødker, 1991; Bertelsen and Bødker, 2002). Robinson (1993) coined the term 'common artefact' and, using the hotel key rack as a prominent example, identified a specific mediating quality of 'the common'. This quality consists of: 'belonging equally to or shared equally by two or more', 'relating to the community as a whole', being 'prevalent', 'occurring frequently or habitually', and being 'usual' or 'ordinary'. When creating an ediary for workers at a paper mill, Kovalainen et al. (1998) found that achieving success in terms of habitual use of the e-diary was attributed to its being open, but not too open; inclusive, but not closed; covering communication, but not exhausting it. This is a description in line with Strauss's definition of habitual use as creating 'locales'. Fitzpatrick et al. (1995) built on the notion of 'locales' when proposing a groupware system that is designed to 'facilitate an integrated work environment and seamless transition among different work settings' (op. cit. p. 10) via a desktop interface.

Neither Kovalainen et al. (ibid.) nor Fitzpatrick et al. (ibid.) deal with prototypical encounters, but take an already existing social and physical structure for granted. Their design is meant to accommodate situations like the habitual use of space for specific groups of workers, while we are concerned with the preservation of traces among more casual ensembles of work. Still, their work demonstrates the importance of paying attention to how the artefacts of our work environment afford preservation of social traces and the experiencing of such social traces.

Numerous authors have addressed the issue of distance cooperation between permanently distributed groups, from Bly et al. (1993) to works like Begole et al. (2002). Many of these proposals make use of video, and some of the more recent ones also include instant messaging (Nardi et al., 2000a and b). The authors often address the habits and rituals arising in distributed groups when the groups gain access to each other through new mediators. 
Dourish and Bly (1992) point out how the everyday contact among physically distant colleagues increases simply because people can initiate communication when they know that their distant collaborator is present. At the same time, video contact gives rise to various problems. It is not always evident who your viewers are, for example, or whether you yourself wish to be constantly visible when you are in a particular location. First and foremost, video is often presented as a substitute for direct physical contact, rather than as a means of leading up to an interaction.

McCarthy's UniCast, GroupCast and OutCast applications (McCarthy, 2003) are concerned with some of the same questions and ideas as we are. UniCast is an individual extra surface where peripheral applications may be run; GroupCast is a version through which group members become aware of, and familiar with, each other, as Web sites and other items of common interest appear on the GroupCast screen. OutCast gives the owner a way of showing outsiders who he or she is. UniCast seems to be used mainly as supplementary screen space to run additional utilitarian applications such as traffic information. GroupCast, on the other hand, is intended to allow group members to get to know each other by showing, say, that several groupmembers use the same (non-work) Web sites. This, we believe, stands in contrast to the desire observed in several groups in our case material to retain and display who they are and what they have been working on together, as in example 2 and 3.

The tension between awareness and ephemerality is addressed by Clark (1996), who argues that a 'common ground' is a linguistic precondition for mutual understanding. Our interpretation of his argument is that a trace of human activity is recognized as 'social' when it allows someone to acquaint themselves with others without receiving explicitly expressed information about them. This is different from an application like OutCast, which projects your own chosen representation of yourself to the outside world. This could also reflect a difference in culture between Europe and the USA similar to the difference regarding homes discussed by Bell et al. (2005).

To sum up: When we confront our findings in case studies of flexible work with the findings in CSCW literature, we find that more research is needed regarding the interplay between ephemerality and continuity in supporting social awareness. We find it important to further investigate ways to leave and recognize traces, and to leave "breadcrumbs" as tokens of other people's actions and existence. Such breadcrumbs should not be so dominating as to call for focal attention; nor should they be so inconspicuous as to slide out of focus. Breadcrumbs can only serve as pointers, however, when other people interpret them as such. Awareness takes two actors and a medium, as well as a place to sprinkle the breadcrumbs so they are likely to be found. We have used this model in a number of explorative design processes in order to 
investigate the question of which structures to support to facilitate social awareness in flexible work, as discussed in the following.

\subsection{RESEARCH METHODOLOGY}

The Center for New Ways of Working, located at the University of Aarhus in Denmark, has the triad of physical space, organizational structures, and computer support as its integrated object of study. In particular, the center is concerned with knowledge sharing and learning in connection with the 'New office.' Prior to the work presented here, and as already mentioned, we and our colleagues have conducted field studies, some of which involved participatory design and the facilitation of change processes (see, for instance, Christiansen, 2001; Bjerrum and Bødker, 2003; Aaløkke et al., 2005; http:// www.nwow.alexandra.dk).

The focus on social awareness originated from these studies. More specifically, our design orientation is motivated by certain findings resulting from the studies mentioned above.

Our research process consists of three elements: gathering and analyzing empirical field data (as described in Bjerrum and Bødker, 2003; Aaløkke et al., 2005); exploratory design processes; and deconstructive analysis of concepts and findings. We have already illustrated this long with our theoretical/conceptual work.

Going into greater detail, the three above elements in this exploratory research-by-design process consists in the following:

1. We have used our own open office setting for exploratory purposes as a microcosm (Engeström, 1987). This microcosm office has five to six people working together in an open space. They are researchers and teachers/consultants. To some extent they have shared tasks and activities, but they are not part of a project team. Everyone has activities outside the office: some have days when they work from home while others have periods of intensive fieldwork. They do not 'own' a specific desk, but move on a regular basis. They have access to an adjacent room used for reading, telephone conversations, and ad-hoc meetings. They are provided with laptops, WLAN access, cellphones, and access to an array of experimental technologies.In this office we experienced working in a flexible environment. We drew on evidence from our case studies to evaluate the relevance of these experiences, explored various CSCW technologies to understand the possibilities of supporting awareness and cooperation, and used those experiences to raise further questions to the case studies (bringing experiences from the microcosm to more general real-world settings).

2. As described in Bødker and Christiansen (2004), the case findings were used in scenarios of particularly interesting situations and reiterated in the 
explorative design. Scenarios were used to hold on to the main problems as we saw them. A central scenario was formed and reiterated in the interaction in several overlapping groups of researchers, who carried out the field studies and took part in the participatory design. The scenario does not represent particular examples taken from specific work situations, and as such they are of a different nature than the design basis arising from ethnomethodological studies, which are well known in CSCW. Our way of working with scenario construction is characterized by them being constructed in tight loops with their use in groups of designers and users. Just like any other design representation, scenarios serve the double purpose of bringing about the decisions made in the design situation and of being a medium of communication between the persons involved in the design activity. Because scenarios are used as boundary objects in multiple prototyping experiments, they support the negotiation and boundary understanding of design ideas, rather than one or more solutions. In this way the scenario maintains its own ephemerality and prototypicality, as discussed in Bødker and Christiansen (2004).

3. Artefact-driven exploration is our way of seeking and finding new knowledge in design. We use prototyping processes to help us understand the possibilities and problems of a background technology, and particularly to determine what questions to ask these technologies. As discussed, we view both the social practices of the new office and the situations that we are examining specifically by virtue of their prototypicality. If one is to investigate technologies and social situations in the making, it is not sufficient to start from current practice alone. Hence, we have been prototyping not to create solutions per se, but to explore and question the context of future design solutions. Our prototypes have been used to provoke social innovation and to try out ideas. Accordingly, the inhabitants of the microcosm office are not seen as end users of a technology developed in an iterative design process, but as collaborators in the exploration of various hypotheses (see Bødker and Christiansen 1997).

The fact that we have used our own office space as a microcosm for experimentation has, of course, been subject to self-reflection from the very start. The habitual use of the flexible office has developed through a process including regular office meetings where problems are raised and discussed. The prototyping experiments aimed at finding potentially new ways to cooperate in the workplace have evolved as a Schön-inspired reflection-inaction (Schön, 1983), and as research they indeed beg the question: How do we know that the experiences gained in a small office occupied by six researchers can be translated to a larger office setting with 30 tax officers or 100 software engineers? Well, in some ways we do not, and cannot, know, but ultimately we are doing the prototyping to find inspiration for further 
prototyping in "real" settings. At the same time, the profiles of these researchers do put them on a par with many other groups we have studied: the amount of time they spend in the office is comparable to other settings, as are their patterns of cooperation inside and outside the office.

In this way of doing research, the exploratory design process serves to probe questions of possibilities and limitations rather that suggesting closed solutions. Furthermore, the prototypes were developed in very close cooperation with the users of the office, and the ongoing self-reflection mentioned above resulted in an extremely close integration of design and use, even going so far as to include participatory design. The prototyping processes themselves have been documented in pictures and field notes, and in some instances captured on video.

The current status of our experiments is as follows: In conjunction with presentations and workshops in various case settings, we are using the video prototype and other prototyping material to seed discussions on future social-awareness technology. This enables us to gather material for a new round of experimentation with such technologies. Many cutting-edge technologies are becoming available, and new experiments will naturally have to include the most recent innovations, as well as being linked to the supplementary case material we continue to assemble as part of an ongoing process.

\subsection{PAPER OUTLINE}

Our compilation of theories, empirical studies of office settings, general experiments in our own open office, and the three prototyping activities presented here have been carried out in a highly iterative manner over an extended period of time. In contrast, the presentation itself is linear. In the introduction we argue that it is relevant for CSCW designers to pay attention to structures that support social awareness in flexible work, and we introduce our germ-cell conceptual model comprising 'breadcrumbs' left and found/ interpreted via a 'shared mirror'. In Section 1 we further account for the basic elements of our research. Section 2 deals with design. We describe how we have detailed our understanding of social awareness in order to be able to subject it to design experiments, dividing this awareness into signs of relatedness, belonging, and care. This conceptualization we anchor in CSCW theory concerning flexible work, social awareness, and relationships between the common, the private, and the public. Following this, we describe three consecutive prototyping processes, since in our experimental setting we have confronted our conceptual model with practical experiences and prototypes. In Section 3 we discuss insights from the prototyping experiments regarding social awareness in flexible work. We focus on how social awareness can be supported by CSCW applications that support structure formation in flexible work. Furthermore, we discuss the effects of our investigations on our future work in the field of $\mathrm{CSCW}$, with regard to both technologies and concepts. 


\section{Exploring social awareness structures}

The review of CSCW research on social awareness and the conclusion of the field studies of Bjerrum and Bødker (2003), Christiansen (2001), and Aaløkke et al. (2005) lead to an understanding of social awareness as a lateral aspect of awareness, a phenomenon to which researchers have access only indirectly, through the analysis of space, mediators, and human conduct and culture. Because it is lateral, social awareness does not meet the eye, nor can it be precisely measured. It must be experienced, in a phenomenological sense, and must hence be accessed by applying a phenomenological approach. Initially we defined social awareness by inverting the threat that Sennett and other sociologists have described as 'corrosion of character' and the process that leads to this effect. We found that the opposite, 'building character', is produced by individuals feeling that they belong, that they are one of a kind, and that they are responsible and care. As a first approximation we decided to understand social awareness as a conscious feeling of belonging, relatedness, and care prompted by the environment. We then began to look for metaphors that might guide our design ideas in this direction.

Following McCarthy and Wright (2004), who look at the processes of experience from a pragmatic point of view, we searched for indexes that comprise the sensual, emotional, compositional, and spatio-temporal focusing on the notion of 'common' (Robinson, 1993), and the border work between the private and the public (Dourish and Palen, 2003). McCarthy and Wright (2004) build on Bakhtin's work when they focus on the relationship between the self and others as a three-part relationship encompassing "how I see myself", "how I see others", and "how others see me". From this we inferred that for social awareness to be prompted 'I' must have the opportunity to be reflected in my environment, and ' $\mathrm{I}$ ' must be able to see how others are reflected, just as they must be able to see the reflections of 'me'. In a sense, then, we have to share a mirror. This resonates with Denning's idea (Denning, 2003) that people must be aware of each other to be capable of committing themselves.

The idea of reflection - of coming back to 'me', and back to 'you' - led us to examine the concept of 'home'. Phenomenologically speaking, my home is the place I return to, it is here I deposit tokens and trophies from my voyages, it is from here I set out into the unknown (Tillich, 1987). We also use 'home' about behavior that we feel comfortable with, and about our own culture.

When on the move, however, as we are in the case of flexible work, we can loose our way back home. It was this observation that led to the "breadcrumbs' metaphor. We recalled how, in the fairytale of Hansel and Gretel, the children manage to keep from getting lost by dropping a trail of breadcrumbs to mark their route - allowing others (their father) to find them, and enabling them to find their way back home. Following this trail brought us 
full circle, and we found ourselves facing the metaphorical "shared mirror" surface. In this second metaphor, supported by the breadcrumbs, we found reflected the three qualities of social awareness that we decided to support by design:

1. Relatedness has to do with movement in space and can be experienced on the borderline between communities of practice, between the virtual and the physical; it is a matter of proximity in both mind and body. It is emotional. You move closer or farther away depending on the attraction, and the attractions as well as your movements are culturally interpreted. Basically, relatedness is a matter of negotiation between meaning and experience.

2. Cognitively, belonging refers to a classification and an object relationship. My sense of belonging is experienced as an expansion of myself into a sphere outside me, with a simultaneous acceptance of something outside me invading and taking up space inside me.

3. Care is a deed, a lateral aspect of certain actions experienced as a culturally mediated attribution of meaning to any mundane act. An action is experienced as care if its meaning comes from the receiver: I can give you a piece of cake, but you decide whether my action is caring, or is an attempt to make you fat and ugly. This way 'care' becomes boundary work, too, because the initiative resides with the actor even while the sense-making resides with the receiver.

In the following we present three exploratory design proposals and research-by-design processes, all built on the reflections presented here, and all analyzed with respect to how the prototypes accommodate and mediate social awareness, understood as the experience of relatedness, belonging, and care.

The three design proposals are: 1. Itineraries, public, private, and common; 2. Personal panels; and 3. Ambient awareness cues. All three build on the metaphor of a 'shared mirror' and the idea that people leave and find 'breadcrumbs' there. This way the prototypes address boundary work between the private and public spheres, between now and forever, between individual and group, and between practical problem-solving and lateral experiences.

\subsection{SITUATION AND SCENARIO}

The scene that we have been exploring in the physical space is one in which a small group of people works in an open office. They are all provided with laptops, WLAN access, cellphones, and access to an array of experimental technologies, including electronic boards and wall-mounted displays. They have access to a shared calendar system, e-mail, BSCW, and so on, including virtually any technology that one might desire for the sake of the experiment. 
Technologically, the actual situation is richer than most of the offices in our case studies.

This scene is a reflection of our own microcosm. The actual group of users/ designers is a small group of researchers and teachers/consultants who work as described above. The situation also reflects problems and challenges from our field studies in other organizations, as previously indicated. This was the starting point of the scenario that was developed and reiterated in the research-by-design process (Bødker and Christiansen, 2004). The prototyping processes discussed in the following were carried out in our own microcosmic office, in collaboration with its other inhabitants, who served as co-designers. Accordingly, the references to "we" in the following summary of experience, refer to the joint reflections of the entire participatory design group.

\subsection{PROTOTYPING 1 - ITINERARIES}

The initial claim of the first prototyping process is as follows: Co-workers, other office inhabitants, and visitors need varied access to the itineraries of the office's inhabitants. A shared calendar provides very fine-grained information of this nature. However, our hypothesis was that office inhabitants do not necessarily wish to expose this level of information to people who happen to pass by their office, or even to all of their officemates. It is not obvious that people who come to look for a particular inhabitant need the level of information given through the calendar, nor that the calendar provides the overview necessary for officemates to answer telephones and so on. The overall idea was that there could be more than one overview of the itineraries (say one on the door and one in the room), and that these could be updated

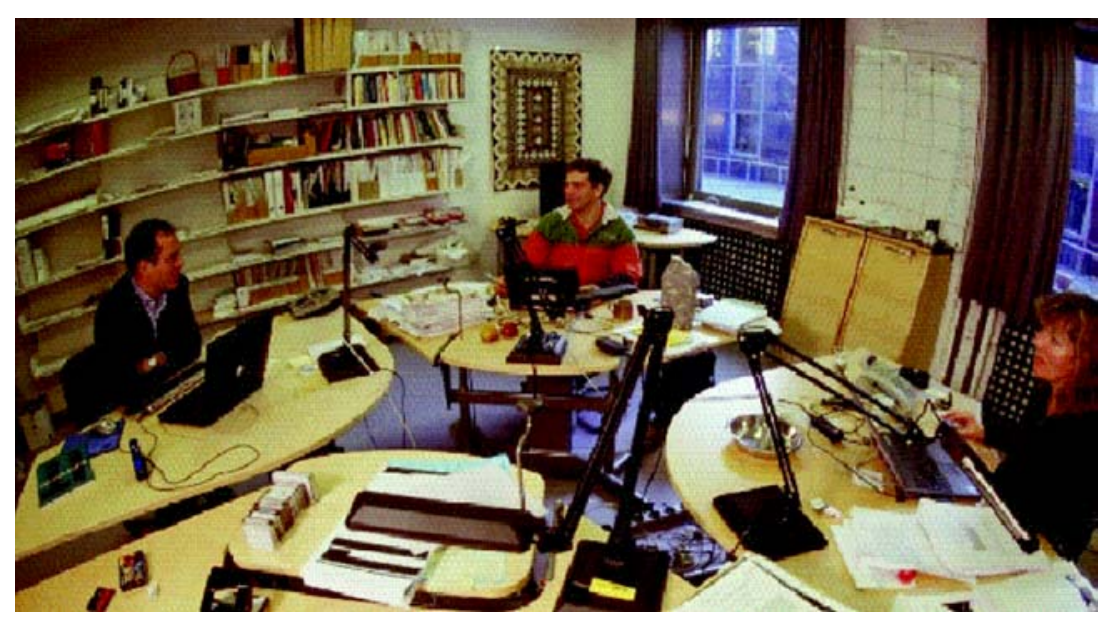

Figure 1. The open office. 
simultaneously, from the office or even from a remote location. You want to inform people about your whereabouts, but you may prefer to display yourself differently to different groups of people. (see Figure 1).

A process was initiated in which a two-level whiteboard calendar was set up for the microcosm office: In the hallway the inhabitants would post information for visitors and visitors could place notes for the inhabitants. Inside the office, a more detailed itineraries list was set up to give an overview of where all inhabitants would be during the coming week/month.

Data was gathered from the use of the boards for a month in the form of daily pictures of the two boards. The researchers analyzed these pictures, with focus on recurring patterns of postings and messages. In addition, we carried out experiments involving the physical location of boards in the room (both inside the room and in the hallway). This was done in a participatory design workshop with all inhabitants.

We found that people maintained the shared itineraries for the sake of the group (in this case it was clearly not a management requirement). The types of notes posted in the hallway were different from those in the office - not only were they shorter, they were also targeted at public inspection. Figure 2 shows one iteration of the hallway board. The board has two fields, one labeled "Present?" and the other labeled "Messages." One inhabitant has written, "I'm here", another: "Back Wednesday" For a third inhabitant, information has been added in two steps with different colored pens. As for messages, a visitor has written to one inhabitant: "Ole, I'll come back to look for you (signed)". This message is fairly typical of those written in the onemonth trial period; rather imprecise indications from someone that he or she was there, will come back, and implicitly, that the office inhabitant may want

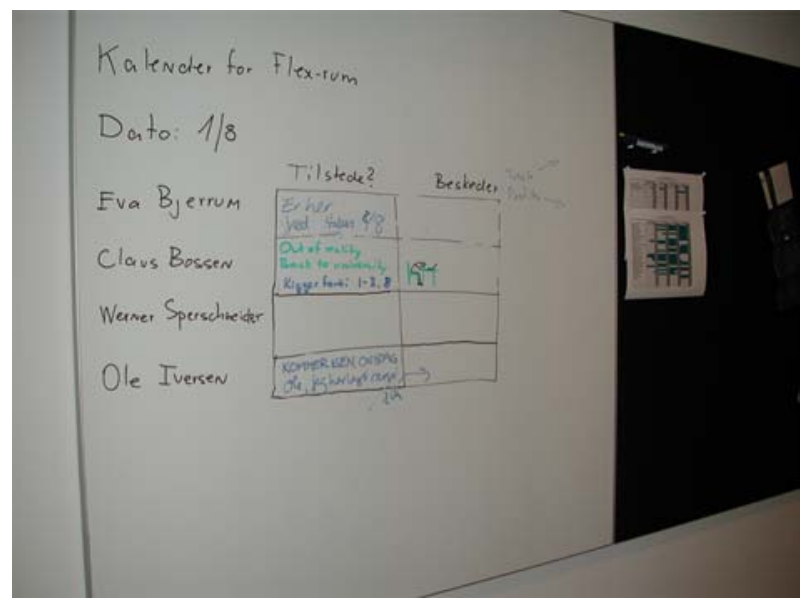

Figure 2. Hallway board. 
to look for the visitor in his or her office, whenever appropriate. The hallway board contained notes addressed both to outsiders and to the group (from inhabitants to visitors and vice versa). In the terms of Moran and Anderson (2000), the social interaction includes interconnections of varying complexity (ranging from messages to particular outsiders, or from inhabitant to visitor or vice versa, to one-to-many interconnections within the group or meant for a less well-defined outside world).

In contrast, the internal office board was structured to show four weeks. Each inhabitant wrote information that ranged in granularity from a particular activity carried out by that particular person, on a particular morning or afternoon, to information that needed to be interpreted in context, such as "home", "fieldwork", or a large cheerful sun indicating "vacation".

This experiment extended Grudin's 'privacy and experimentation' concern into the social domain. It gave a different view on the 'degree of engagement' than the one presented by Moran and Anderson (2000). Their concern is with 'peripherality' as a lead into direct engagement, whereas here the difference between being peripheral and central is mainly related to different types of social contract. An essential issue relates to the pragmatics of the interaction (Moran and Anderson, 2000): The idea of shared itineraries illustrates how it is necessary to aim for a design that enables users to view different levels of planning information, instead of giving universal access to a shared calendar. How well do people need to know each other in order to interpret plans as 'breadcrumbs'? Or, in the terms used by Clark (1996), how much 'common ground' is needed? The difference between what was actually written inside and outside the office shows something about the expectations of inhabitants and visitors alike. As for the hallway board, inhabitants expected that visitors would want to know when inhabitants would be in their office next, but no detailed plans. Similarly, visitors mainly left casual notes indicating that they had stopped by. The internal office itinerary provided information about four weeks, with details of where and when to find people. Phone numbers were left at which people could be reached, leaving far more explicit 'breadcrumbs' than in the hallway. The discretionary power to make distinctions in many ways rested with co-inhabitants: Could an officemate's home phone number be given to a caller? Probably so, if the person was working at home, but less likely if the person was on vacation. Dourish and Palen (2003) discuss how individuals create privacy by constructing their appearance on the Internet. Similar issues are at stake here, and not only for individuals: Individual inhabitants need to construct their own appearance within the office as well as externally, as does the group itself, particular in relation to the outside world. This is an example of boundary processes that take place at personal and group levels. It is also a strong indication that not only individuals, but groups, too, need to experiment in private (Grudin, 2002). Furthermore, it seemed important to be able to produce the bulk of this information only 


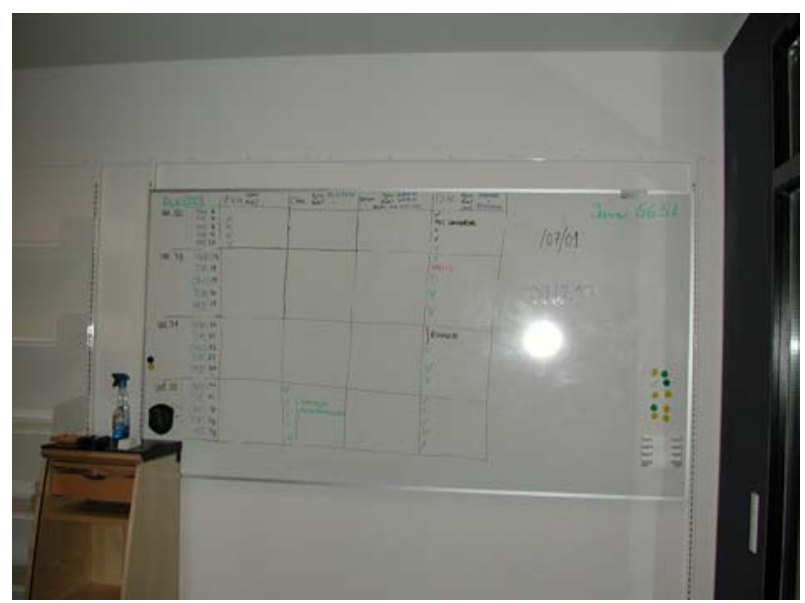

Figure 3. Room board.

once as a least-effort strategy for all those involved (and also, for instance, to use this to generate "out of the office" e-mail messages and voice-mail replies). On the face of it, these two requirements seemed contradictory.

Accordingly, the main open-design question is how these two sets of requirements can be combined in a flexible way, meaning how can one obtain different granularities of itineraries for different places while at the same time ensuring that the itineraries are updated only once? Robertson (2002) points out how different awareness cues are learned. In addition, we point out that cues offer different learning potentials for group members and for casual visitors. There is, furthermore, a need for tailorability, since the appropriate granularities and habits cannot be established once and for all.

With respect to the three experiential questions about relatedness, belonging, and care, the participants found relatedness expressed in being named on the board, in receiving messages, and in others seeing that you receive messages. The sense of belonging was experienced through the commitment to maintaining the itineraries, and the other members' commitment to the group was measured on the effort they put into feeding in information. Accordingly, although it is desirable to have factual information, such as calendar information, created only once, it is less desirable to have this undergo an automatic transformation in order to be placed on the boards. There is an inherent tension between how to construct one's appearance to co-workers and outsiders and one's appearance to others. Traces of informal, nonsensical or humorous messaging were interpreted as tokens of care, and as a sign of the existence of a 'commons', governed by rules growing out of interaction between the inhabitants, and based on a growing sense of 'we'. Since the learning potential is larger among group members than between the office inhabitants and outsiders, a further 
distinction influences the need for a technology that does not assume the two types of information to be identical.

\subsection{PROTOTYPING 2 - PERSON PANELS}

Prototyping process 2 is based on the following claim: In open offices, there are often no means of 'leaving stuff around' and creating a look and feel of yourself or of a group (see Bjerrum and Bødker, 2003). The lack of historical documents makes it difficult to leave and come back, and makes it difficult as well for others to recognize the presence of someone who is not actually in the room.

The examination of this hypothesis mainly took place in a participatory design workshop with office inhabitants in their open office. Through handson experiments with mock-ups of alternative solutions, the participants coconstructed a set of pros and cons for the alternatives, and this constituted the basis for our further theoretical elaboration.

Specifically we aimed at creating personal places through electronic panels. The general idea was to provide some kind of electronic panel at each desk where personal 'stuff' would pop up in a non-intrusive way whenever someone was occupying the desk. This additional panel was intended to offer space for posting electronic sticky notes and other reminders, as well as for children's drawings, family pictures, and other personal items. We explored three different solutions: panel, desktop, and wall displays (Figures 4, 5, and 6 ). These turned out to have different potentials and problems with regard to personal and shared access.

We concluded that the wall (Figure 5) was the most communal of the three solutions, but the one least supportive of individual user attention, for instance being the least suited for posting sticky notes. The wall did, however, work well for shared project spaces.

The screen embedded in the desktop (Figure 6) worked well for personal use, but was more problematic in supporting awareness in passers-by. The panel rising from the desktop ("screen no 2" in Figure 4) had qualities that lay somewhere between the other two solutions, being within view of the user and visible to people passing by or seated in other parts of the office.

Personal panels certainly could not replace piles and artefacts on the physical desktop, although the panels did seem to help inhabitants leave trajectories of current and pending activities and return later. Furthermore, people could leave trajectories of past activities as reminders to themselves or others, and they could bring up more personal items, like family photos. Three advantages in this solution as compared to bringing up this kind of information on the main computer screen were: (1) avoiding clutter on the screen; (2) allowing the laptop to be carried to meetings, and content to be projected onto a wall without exposing personal items; and (3) enabling 


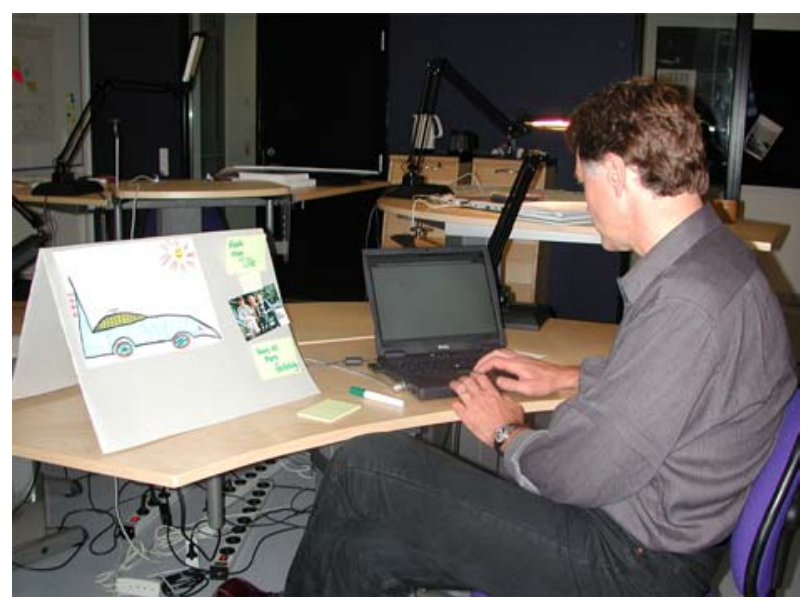

Figure 4. Screen no 2.

officemates to peripherally survey the traces without looking over the shoulder of the person working (allowing experimentation in private). Regarding groups, a wall-based version of the panels would serve similar purposes, reminding the group and others of the group's identity and members, of what it has achieved in the past, and of what it is working on. These prototypes are based on some of the same ideas as UniCast, GroupCast and OutCast (McCarthy, 2003). GroupCast is meant for group members to get to know each other. This, we believe, stands in contrast to the desire expressed by several groups in our case material to hold on to and

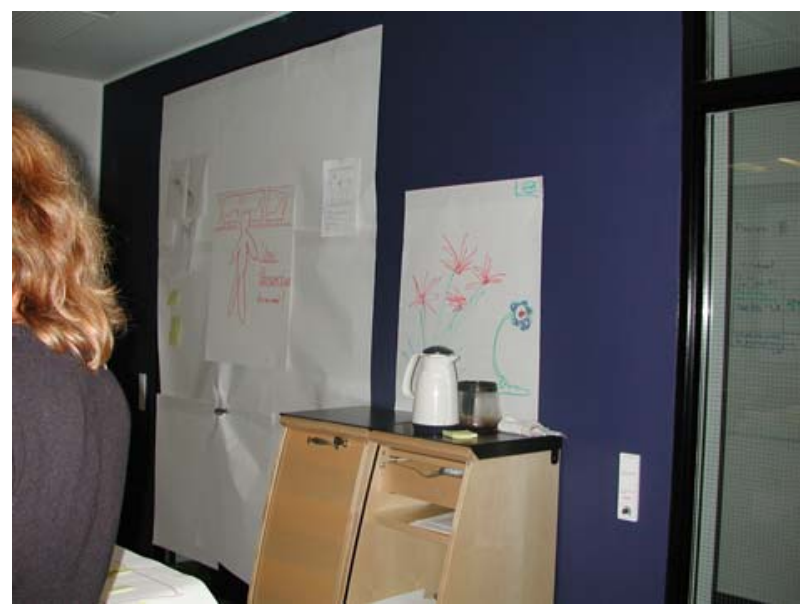

Figure 5. The wall. 


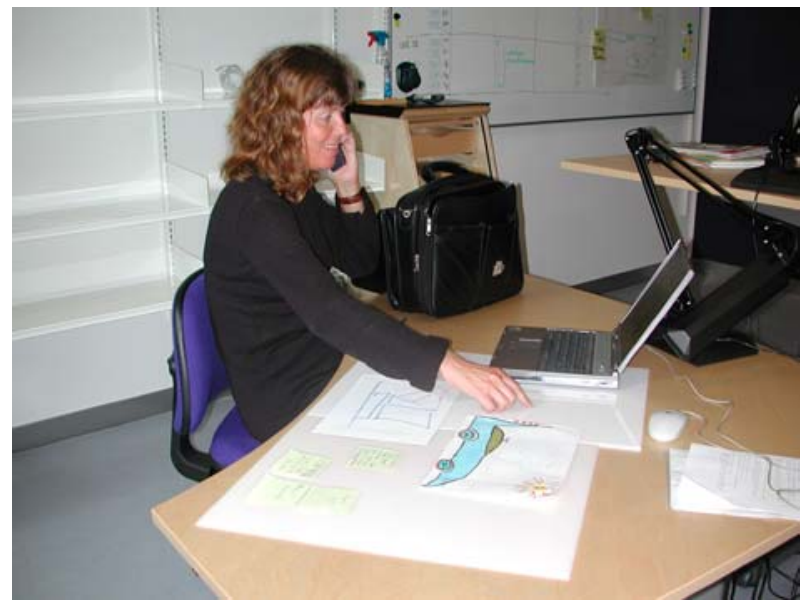

Figure 6. The desktop.

show their common identity and what they have been working on together; to retain a 'shared mirror'.

The form our proposal took made it possible to discuss, among the microcosm office inhabitants, the degree of privacy of information in relation to which type of panel they preferred. These discussions raised questions about the tension between traces and privacy. For instance, how much of what is left behind should also be visible to others? There are further tensions

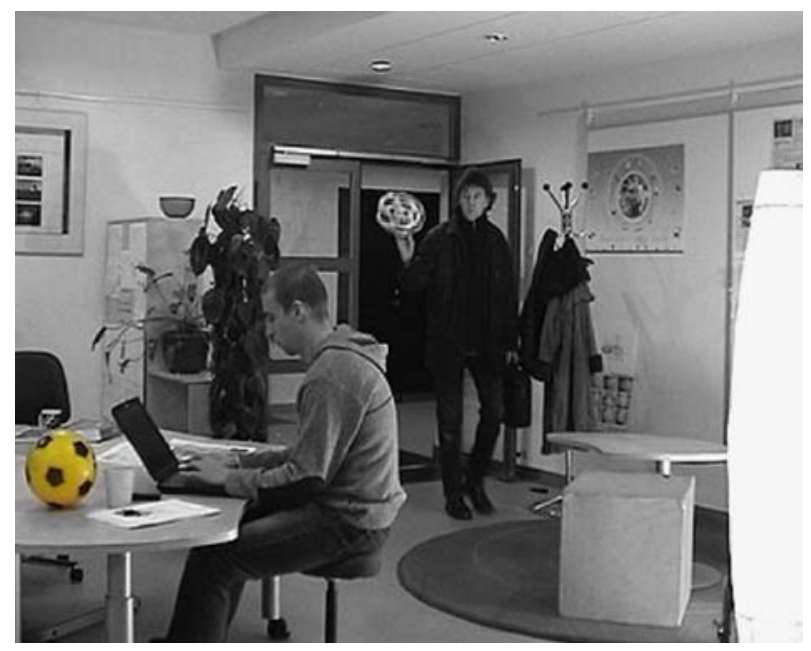

Figure 7. Combining physical and virtual cues. The ball is the cue for one person; the bike/bike helmet for another. The cues turn up as well as the two people's virtual presence. 
between the immediate working materials needed to do a job and the background material that may be needed for one to feel at home in the office. Technically and socially it is a challenge to know when, and for how long, a particular person has occupied a desk. It obviously seems like at good idea to assume that whenever someone places a laptop on the desk, that desk is occupied. However, it is less obvious what happens when someone else comes over and places their computer next to the first. And the mere fact that a laptop has been removed from a desk is not necessarily a sign that the person has left. The experiments indicate a need to further explore the implications of different design choices to form a social order in the office: What does it mean to claim a desk? When can someone else take over the desk, and when are they only spending a moment there visiting or working with the person who has already claimed the desk?

The participants found that the effort to use a panel in addition to the computer was experienced as relatedness. The semi-public information was seen as an invitation to participate. However, if personal 'stuff' is put on display and no one else notices, it makes people feel excluded. Using personal panels offers a way for co-inhabitants to show that they care: All they have to do is pay attention and ask a question or two. It accordingly makes sense to avoid automatic log-on and log-off of these panels, since the personal items must remain there for inspection when the occupant is not actually at the desk.

\subsection{PROTOTYPING 3 - AMBIENT AWARENESS CUES}

Our third claim is that participation while away and learning about other people's habits is particularly problematic when hot-desking. Hence, we looked for ways to create ambient awareness between the inhabitants of the office while some of them are away. According to Brown and Duguid (1994), it is the traces you leave while choosing one trajectory instead of another that make this specific trajectory more compelling to the next person. When the next person chooses the same trajectory, this person adds traces that eventually will turn the trajectory into a locale. This adds a habit to the culture and thereby supports community-building.

In this case the design team chose to make an advanced video prototype in collaboration with inhabitants of the microcosm office. The inhabitants acted out their everyday work situation in a scenario emphasizing hot-desking, distance, coming and going (Bødker and Christiansen, 2004). Through this process they explored the possibilities and problems of a technology that would help them leave and trace physical and virtual 'breadcrumbs'. Video was used to capture decisions, and ultimately a video demonstrating the design result was put together. This video prototype has been used to discuss possible design alternatives with a number of audiences, from designers of awareness technology to people working in hot-desk settings. 
The idea agreed upon by the joint design group was to investigate the possibilities of seeing who was "present" in the room through 'breadcrumbs' as a combination of physical traces and virtual equivalents, rather than through very bold and direct signs. Further, we wanted to allow the virtual side to be visible to people working at home, for instance, and at the same time to allow them to show their virtual presence in the office - through the 'shared mirror'. A coffee cup could indicate the officemate who normally made coffee in the morning, say, or a person who is very fond of coffee, and a bike helmet could indicate someone who always rode his bicycle to work. Physical habits and preferences served as the starting point, thus supporting people in getting to know each other, and in leaving traces that were primarily intended for their officemates.

One of our ideas was that it is necessary to learn about each other's habits in order to learn to recognize each other in the virtual environment. The video prototype, however, ran into problems when trying to tell this story through subtle relationships between physical habits and virtual ones, and the design remained mirrored down in fairly direct iconic representations. Based on the reflections of the office inhabitants, we conclude that a further focus on ambience is needed.

Outside the office, the presence of workers who were physically present in the building was shown through their icons. For absent co-workers there was an indication of when they would be back. It was our collaborative decision that physical presence is not a matter of whether a person is actually in the room at a given time (something easily ascertained by glancing around), but a matter of whether they have established themselves in the office by placing their working materials and leaving physical traces.

This exploration raised additional questions regarding the granularity of information about the activities of others, particularly when the video prototype was contrasted with a shared electronic calendar: What does it mean to be 'present', and how does one leave traces of presence in the virtual world? What are the relationships between habits in the physical and virtual world, and how does one learn from subtle cues that bridge the two worlds? And not least, how can these new cues be learned (Robertson, 2002), and how do new social relationships develop, on the basis of these new cues?

This investigation raised particular socio-conventional questions concerning the status of people working from home: When are they working at home and when do they wish to be considered as "working" and accessible in the shared (virtual) space? Alternatively, when have they just switched on their computer to order a movie ticket? What if they want to check their e-mail without being disturbed? This exemplifies a situation where someone may wish to remain peripheral to the social situation at work, and wish to avoid being "dragged" into a sphere of activity. Through our joint discussions we found that it is probably necessary for people to sign on explicitly to the 
shared space from home, even though this results in certain overheads that are not otherwise there.

This investigation is not about distance-cooperation between permanently distributed groups, a topic dealt with by many authors in CSCW, including Begole et al. (2002). Accordingly, the findings do not quite compare to studies in which the shared computer technology is the one main source of communication and personal familiarization. However, the experiment was too limited to yield any long-term findings with respect to the three experiential questions of relatedness, belonging, and care. Discussions of the prototype with various audiences make us point out that creating one's presence in the virtual workspace by means of an object one cherishes is very much making a home - you take up space and attention simply because you are you, and you are there. This act of conquering space is fundamentally social, as it is a negotiation of power over space. Thus, when signing on as, say, a football, you get a sense of relatedness (to those who may sign on as a flower or a mug), a sense of belonging (since you take the trouble to claim a space there), and a sense of care (since others recognize you as a person, not only as a unit of labor).

\subsection{SUMMARY OF FINDINGS}

Through our exploratory prototypes we found that technologies such as personal panels seemed to help inhabitants leave traces of current and pending activities to which they could return. Furthermore, people could leave traces of past activities as reminders to themselves or others, and they could display more personal content, such as family photos. The idea of shared itineraries illustrates how it makes sense to aim for different levels of information about plans - different sorts of 'breadcrumbs' - instead of giving universal access to a shared calendar. If we relate these findings to those of the CSCW literature, it is possible to find inspiration for various designs and ideas on how to proceed.

There is, however, an area of conflict tension in our findings that is most often not dealt with. The technologies that we design often place themselves in the foreground of our attention and awareness: Technologies help people stay in contact once they have created an awareness of one another, and the technology offers itself as a recording and reporting device. Technologies that offer the level of background information that we have been looking for are rare. Furthermore, there is a self-contradictory element in the design processes itself: We design to put our technologies in the background, but when explored by users they are often in the foreground simply because they are new. In actual fact, emergent situations are difficult to deal with in design.

It is nevertheless possible to step closer to the technical challenges clarified in our investigation. We have raised questions about the granularity of 
information on other people's activities: What does it mean to be 'present', and how does one leave traces of one's presence in the virtual world? What are the relationships between habits in the physical and the virtual world, and how do we learn from the subtle cues that bridge the gap between these two worlds? How can these new cues be learned, and a 'shared mirror' created?

The examination of the prototyping experiences has helped us to become more specific about the challenges we are facing.

1. Creating a presence of, and a presence for, others: First of all, icons or symbols of others need to stay in the background and be non-intrusive. Secondly, user sign-on/sign-off cannot be entirely automatic, and gives rise to technical and socio-conventional challenges. Only a given user can decide whether he or she is to be considered "on-line" when merely checking the weather forecast or ordering a movie ticket, or conversely "off-line" when leaving a desk or moving a computer.

2. Communicating one's movements and plans: The boldness and detail of the information must be balanced with the needs of the receivers. It should be possible to ignore such information, and it is rarely in the interest of the receiver to have to interpret the details of someone else's calendar. In addition, personalizing such information can be important, depending on the joint habits in the office.

3. Constructing a home when hot-desking: People need to be able to put personal items, their own "stuff", on display as an invitation, without any obligation to comment. Rather than being a series of personal emblems the user carries around, this "stuff" is something the user must be able to leave behind, for others to inspect in privacy.

4. Leaving traces: For the individual, too, it is essential to be able to leave traces of past activities to return to at a later date, or even when moving to a different desktop.

5. Creating a common space: The growth of a common space with common rules is a process that begins with a commitment to maintaining input, and a commitment to reading and commenting on output related to personal 'breadcrumbs'. An environment that affords opportunities for non-rational information activity can trigger such a commitment.

\section{Social awareness and care rationality}

In this section we will return to the CSCW concepts and solutions presented above in order to clarify the significance of our prototyping processes for designing solutions that support social awareness.

Many attempts to support social awareness with computers can be seen as attempts to support existing communities, either by augmenting the community or by substituting the physical meeting places with virtual ones. In our design experiments we have not taken an existing community for 
granted, but targeted support of community structures in the making. Accordingly, our focus is on traces and mirrors rather than on fixed boundaries.

An example illustrates the difference: In several instances, such as Babble (Erickson and Kellogg, 2002), the authors discuss the problem of newcomers entering the system, and of regular users not knowing who these newcomers are. We do not address this as a problem to be eliminated, but as a chance to build community. We are therefore concerned with how a newcomer, under the care of old-timers, can create traces that will eventually become part of the community, perhaps by pushing the border between tradition and the unknown.

Furthermore, if we take up Grudin's challenge of letting newcomers experiment privately, we must face the tension that exists between direct and indirect information about the activities of co-workers: With GroupCast (McCarthy, 2003), for example, it is easy to decide that Web pages frequently accessed by people reflect a certain interest, and that accordingly, if several users access the same Web pages frequently, they are likely to share this interest. However, there can be many reasons why, say, a newcomer might access certain Web pages frequently while experimenting. And indeed, these Web pages might have no relevance at all in view of expressing who these newcomers are, and how they wish others to perceive them. Furthermore, most people pay visits to Web pages that they do not wish to show to others.

Consequently, instead of trying to make silent behavior talk we have focused on situations where people seem to negotiate or want to negotiate on the boundary between the private and public spheres, between the known and the unknown. We found that through such communication, the private/ public boundary may transform into a 'third' place where private is substituted by personal, and public by common, by way of communication and negotiation.

We propose that our designs have succeeded in bridging the gaps between actors' experience of being physically contained and shown a path for action in the physical space; their experience in the virtual space; and their mental experience of sharing places, mediating instruments, and culture. This threeway mediation provides an overall sense of 'home' as feelings of belonging, relatedness, and care. For an explanation we turn to the theory of cognitive semantics proposed by Lakoff (1987). He argues convincingly that interpretations are rooted in a metaphorical mapping from physical space into conceptual space by way of preconceptual structures, of which the kinesthetic image schemas of container, path, and link, seem the most powerful.

Looking back on CSCW, the field has developed in many interesting ways. However, our analysis of what is needed to facilitate awareness points us in the direction of care rationality - in contrast to which most awareness applications are based on means - end rationality. By way of example, while 
UniCast may add to the users' feeling of being at home, it is still a very functionalistic way of extending their space. These were, more or less, the conclusions on CSCW already in the making in Bødker et al. (1988). Accordingly, it seems that even though the CSCW field has developed significantly since 1988, it has not managed to rise to the challenge posed by different kinds of rationality. In particular, this is the case for the experienceoriented trend that has become popular.

Naturally, only longitudinal cultural studies can provide real-life evidence for the assumptions made here. We find, however, that our microcosm experiments indicate a promising route for further experiments with building 'homes'. The experience of containers, paths, and links in the physical space maps onto the experience of belonging, relatedness, and care in the mental space - all of which sits on the borders and boundaries in the physical and mental space.

Further analyses and design explorations addressing such boundary work are needed, but as already mentioned, it is difficult to create studies that are both innovative and longitudinal when dealing with emerging practice. This is due to the combination of many levels of uncertainty and the amount of work required for making prototypes that one can experiment with in these complex situations. Hence, complementary to ethnographic and ethnomethodological studies that take their point of departure in users' existing practice, design research along the lines presented here is a way of gaining knowledge about emerging social structures.

\section{Conclusion}

We began this paper by asking which structures should be supported. We conclude that the structures to be supported are those that create a mapping between a physical experience of being included, being related, and being responsible for and able to express care for others. We have demonstrated that the metaphors of 'a shared mirror' and 'breadcrumbs' work as an outset for designs that support such structures. We have explored the new flexible working conditions and the possibilities of supporting or preventing social awareness in this type of work. We worked to deepen our understanding of the constituents of social awareness - the kind of awareness that grows from overhearing and overseeing recurring situations - when physical access is no longer a constant option. Based on several rounds of analysis of our prototyping experiences, it appeared that the discriminating difference between awareness in the new and the old setting can be found in a shift in the figureground relationship: The 'old' social awareness is based on conceivable patterns of regularity of time, place, people, and behavior in which people find their bearings. In the 'new' type of social awareness, these bearings become the figure - we communicate explicitly and instantly as to our 
whereabouts, our bearings, and our emotions and feelings, using cellular phones or the Internet, in busses, in chat rooms, and so forth. Social awareness has become dependent on making explicit what was once taken for granted: where we are, what we are doing, and at what time of day. Feedback confirms our existence. The important aspect of a phone message or voicemail may not only be the information conveyed. It is just as much the metamessage that 'I am', and what I crave is the feedback that assures me that I am, because I have received approval of my bearings and thereby a confirmation of my sense of self.

To sum up: Social awareness requires breadcrumbs as tokens of other people's existence. They should not be so dominating as to call for focal attention; nor should they be so silent as to slide out of focus. Shared mirrors help us see and relate to each other as individuals and as groups. Social awareness seems to live in a twilight zone between attention and nonattention, tradition and the unknown. To support ongoing sense-making under these conditions, we find that Robinson's (1993) notion of 'the common' captures the most important qualities of the necessary mediators: They afford an open-ended, non-discretionary use - as in the well-documented cases where waterholes, coffee machines, and copiers mediate social awareness by offering themselves to multiple uses in a non-demanding way. If I know something is private, then I know I am not supposed to look, and if I know something is public, then I may choose not to look. In the 'commons', however, I pay attention because I have to find out whether what is happening concerns me: Do I care? Should I help? Could I ask for help? Is there room for me? Am I allowed to move? Can I change the rules? Answers to all these questions depend on the availability of objects that lend themselves to such interpretation.

We began by assuming that the new flexible working conditions may cause the social structures of work inherited from the industrial-work organization to vanish along with the physical structures of this mode of production. We have faced the challenge of considering how computer support for cooperative work may help to restore social awareness under these altered circumstances. A review of case studies of flexible ways of working, backed up by CSCW research contributions in Section 1, provided for the examination in Section 2 of three prototyping experiments: A shared calendar, personal panels, and ambient awareness cues. These were all under scrutiny to assess their potential ability to carry and support experiences of relatedness, belonging, and care. The development and critical discussion of these experiments in Section 3 evolved around the questions of how people create a sense of presence of (and for) others when they are in and out of the office, how they communicate about their movements/plans and keep in touch when they are in and out of the office, and when they are working from home; how they take possession of a space (construct a home) when they work in varying 
locations; how they personalize their space and leave themselves contextual reminders; how locations relate to whether information is public, private, or held in common; and how persistence of social encounters develops.

\section{Acknowledgements}

The Center for New Ways of Working has been financed through the Alexandra Institute and through CIT/CfPC grant \#223. We thank all our collaborators, most notably Eva Bjerrum, Claus Bossen, Christina Brodersen, Jannie Friis Kristensen, Kaj Grønbæk, Ole Iversen, Peter Krogh, Rune Nielsen, and Werner Sperschneider. Thanks are also due, for inspiring comments, to Peter Carstensen, Anne Bøgh Fangel, Marianne Dammand Iversen, Anne-Marie Kanstrup, Lene Kjelsteen, Leysia Palen, and Thomas Risgaard, as well as several anonymous, and very patient, reviewers of earlier versions of this paper.

\section{References}

Aaløkke, S. and E. Bjerrum (2005): The Office as a Strategic Artifact for Knowledge Sharing? OLKOS Trento.

Aaløkke, S., E. Bjerrum, S. Bødker and A. Bechmann Petersen (2005): Gate Keeping or Bridge Building? - Cooperation, Learning and Boundary Working in a Cross-media Workplace. OLKOS Trento.

Begole, J.B., J.C. Tang, R.B. Smith and N. Yankelovich (2002): Work Rhythms: Analyzing Visualizations of Awareness Histories of Distributed Groups, Proceedings of CSCW 2002, pp. 334-343, ACM.

Bell, G., M. Blythe and P. Sengers (2005): Making by Making Strange: Defamiliarization and the Design of Domestic Technologies. ACM Transactions on Computer-Human Interaction (TOCHI), vol. 12, no. 2, pp. 149-173.

Bertelsen, O. and S. Bødker (2002): Interaction Through Multi-artifacts. In S. Bagnara, S. Pozzi, A. Rizzo and P. Wright (eds.): ECCE 11 - Cognition, Culture and Design Eleventh European Conference on Cognitive Ergonomics. Rome: Instituto di scienze e technologie della cognizione consiglio nazionale delle ricerche, pp. 103-111.

Bjerrum, E. and S. Bødker (2003): Knowledge Sharing in the "new office" - Possibility or Problem? K. Kuutti, E. Karsten G., Fitzpatrick, P. Dourish and K. Schmidt (eds): Proceedings of the Eighth European Conference on Computer Supported Cooperative Work, 14-18 September 2003, Helsinki, Finland, pp. 199-218.

Bjerrum, E. and A.B. Fangel (2004): Ny kontorindretning - et videndelingværktøj eller bare smart? Børsens Ledelseshåndbøger Knowledge Management, no. 5.

Bly, S., S. Harrison and S. Irwin (1993): Media Spaces: Bringing People Together in a Video, Audio, and Computing Environment. Communications of the ACM, vol. 36, no. 1, pp. 28-46. 
Bødker, S. and E. Christiansen (1997): Scenarios as Springboards in Design. In G. Bowker, L. Gasser, S.L. Star and W. Turner (eds.): Social Science Research, Technical Systems and Cooperative Work. Mahwah, NJ: Erlbaum, pp. 217-234.

Bødker, S. and E. Christiansen (2004): Designing for Ephemarality and Prototypicality. Proceedings of the 2004 conference on Designing interactive systems: processes, practices, methods, and techniques, ACM, pp. 255-260.

Bødker, S. (1991): Through the Interface - a Human Activity Approach to User Interface Design. Hillsdale, NJ: Lawrence Erlbaum Associates.

Bødker, S., P. Ehn, J.L Knudsen, M. Kyng and K.H. Madsen (1988): Computer Support for Cooperative Design. In Proceedings of Conference on CSCW, Portland, Oregon, September 1988, New York: ACM, pp. 377-394.

Brown, J. Seely and P. Duguid (1994): Borderline Issues: Social and Material Aspects of Design in: Human-Computer Interaction, vol. 9, pp. 3-36.

Christiansen, E. (2001): Knowledge Management in a Thinking System. IRIS24 2001, Ulvik, Norway, August 11-14, pp. 691-704.

Clark, H. (1996): Arranging to Do Things With Others Plenary Session. Proceedings of ACM CHI 96 Conference on Human Factors in Computing Systems, vol. 2, pp. 165-167.

Denning, P. (2003): The Profession of IT: Accomplishment. Communications of the ACM, vol. 46, no. 7, pp. 19-23.

Dourish, P. and S. Bly (1992): Portholes: Supporting Awareness in a Distributed Work Group. Proceedings of ACM CHI'92 Conference on Human Factors in Computing Systems, pp. 541-547.

Dourish. P. and L. Palen (2003): Unpacking "privacy" for a Networked World. In V. Bellotti, T. Erickson, G. Cockton and P. Korhonen (eds.): Proceedings of the 2003 ACM Conference on Human Factors in Computing Systems (CHI'03), pp. 129-136.

Engeström, Y. (1987): Learning by Expanding. Helsinki: Orienta-Konsultit.

Erickson, T. and W. Kellogg (2002): Social Translucence: An Approach to Designing Systems That Support Social Processes. In J. Carroll (ed.): Human-Computer Interaction in the New Millennium. New York, NY: Addison-Wesley, pp. 324-346.

Fitzpatrick, G., W. Tolone and S. Kaplan (1995): Work, Locales and Distributed Social Worlds Distributed Social Worlds. Proceedings of the Fourth European Conference on Computer-Supported Cooperative Work, pp. 1-16.

Grudin, J. (2002): Group Dynamics and Ubiquitous Computing. Communications of the $A C M$, vol. 45 , no. 12 , pp. 74-78.

Kovalainen, M., M. Robinson and E. Auramaki (1998): Diaries at Work Organizational Culture: Memory and Change. Proceedings of ACM CSCW'98 Conference on ComputerSupported Cooperative Work, pp. 49-58.

Lakoff, J. (1987): Woman, Fire and Dangerous Things, Chicago and London: The University of Chicago Press.

Lave, J. and E. Wenger (1991): Situated Learning: Legitimate Peripheral Participation. Cambridge: Cambridge University Press.

McCarthy, J. and P. Wright (2004): Technology as Experience. Cambridge, MA: MIT Press.

McCarthy, J. (2003): Promoting a Sense of Community with Ubiquitous Displays. In K. O'Hara, M. Perry, E. Churchill and D. Russell (eds.): Public and Situated Displays Social and Interactional Aspects of Shared Display Technologies. The Kluwer International Series on Computer Supported Cooperative Work, vol. 2, pp. 283-308.

Moran, T.P. and B. Anderson (2000): The workaday world as a paradigm for CSCW design. Computer Supported Cooperative Work. Proceedings of the 1990 ACM conference on Computer-supported cooperative work, pp. 381-393. 
Nardi, B., S. Whittaker and H. Schwartz (2000a): It's not What You Know. It's Who You Know: Work in the Information Age. First Monday 5(5).

Nardi, B., S. Whittaker and E. Bradner (2000b): Interaction and Outeraction - Instant Messaging in Action. Computer Supported Cooperative Work. Proceedings of the 2000 ACM Conference on Computer-supported Cooperative Work. ACM, pp. 79-88.

Robertson, T. (2002): The Public Availability of Actions and Artefacts. Computer Supported Cooperative Work (CSCW), vol. 11, no. 3, pp. 299-316.

Robinson, M. (1993): Design for Unanticipated Use. In G. deMichaelis and C. Simone (eds.): Proceedings of the Third European Conference on Computer-Supported Cooperative Work (ECSCW '93). Dordrecht, Boston, London: Kluwer, pp. 187-202.

Schön, D. (1983): The Reflective Practitioner. New York: Basic Books.

Schutz, A. (1962): Collected Papers, vol. I. The Problem of Social Reality. Martinus Nijhoff/ The Hague.

Sennett, R. (1998): The Corrosion of Character. The Personal Consequences of Work in the New Capitalism. New York, London: W.W.Norton and Co.

Strauss, A. (1993): Continual Permutations of Action. New York: Aldine De Gruyter.

Tillich, P. (1987): On Art and Architecture. New York: Crossroads, 81-85.

Tollmar, K., O. Sandor and A. Schomer (1996): Supporting Social Awareness @ Work, Design, and Experience Setting up Encounters. Proceedings of ACM CSCW'96 Conference on Computer-Supported Cooperative Work 1996, pp. 298-307. 